\title{
Effects of Nano-anatase on Spectral Characteristics and Distribution of LHCII on the Thylakoid Membranes of Spinach
}

\author{
Lei Zheng $\cdot$ Mingyu Su $\cdot$ Xiao Wu Chao Liu . \\ Chunxiang Qu • Liang Chen • Hao Huang • \\ Xiaoqing Liu • Fashui Hong
}

(C) Humana Press Inc. 2009

\section{Erratum to: Biol Trace Elem Res (2007) 120:273-283}

\section{DOI 10.1007/s12011-007-8025-3}

The original version of this article unfortunately contained a mistake. The authors' first name and last name were interchanged in the original article. The correct names are shown above. 